\title{
Outcomes of salvage high-dose-rate brachytherapy with or without external beam radiotherapy for isolated vaginal recurrence of endometrial cancer
}

\author{
Shuhei Sekii, MD!, Naoya Murakami, MD, PhD!, Tomoyasu Kato, MD, PhD², Ken Harada, MD!, Mayuka Kitaguchi, MD!, \\ Kana Takahashi, MD', Koji Inaba, MD, PhD', Hiroshi lgaki, MD, PhD', Yoshinori Ito, MD', Ryohei Sasaki, MD, PhD³. \\ Jun Itami, MD, PhD \\ 'Department of Radiation Oncology, National Cancer Center Hospital, Tokyo, ²Department of Gynecologic Oncology, National Cancer Center \\ Hospital, Tokyo, ${ }^{3}$ Division of Radiation Oncology, Kobe University Graduate School of Medicine, Kobe-city, Hyogo, Japan
}

\begin{abstract}
Purpose: This study was designed to retrospectively analyze outcomes of high-dose-rate (HDR) brachytherapy, with or without external beam radiotherapy (EBRT), in patients with vaginal recurrence of endometrial carcinoma, and to identify factors prognostic of patient outcomes.

Material and methods: The medical records of all patients who underwent HDR brachytherapy for initial recurrence in the vagina of endometrial cancer after definitive surgery between 1992 and 2014 were retrospectively reviewed. All patients underwent either intracavitary brachytherapy (ICBT) or interstitial brachytherapy (ISBT) with or without EBRT. Late toxicity was graded using the EORTC (LENT/SOMA) scale, revised in 1995.

Results: Thirty-seven patients were identified. The median follow-up time was 48 months (range: 6-225 months). Of these 37 patients, 23 underwent ICBT, 14 underwent ISBT, and 26 underwent EBRT. Tumor size at first examination of initial relapse was significantly larger in the ISBT than in the ICBT group. The 4-year respective overall survival (OS), local control (LC), and progression-free survival (PFS) rates in the entire cohort were $81.0 \%, 77.9 \%$, and $56.8 \%$, respectively. The interval between diagnosis of first recurrence and radiotherapy ( $<3$ months, $\geq 3$ months) was a significant predictor of LC and PFS. OS and LC rates did not differ significantly in the ICBT and ISBT groups. Two patients experienced grade 2 rectal bleeding, and four experienced grade 2 hematuria. No grade 3 or higher late complications were observed.

Conclusions: Salvage HDR brachytherapy is an optimal for treating vaginal recurrence of endometrial carcinoma with acceptable morbidity. Early radiotherapy, including brachytherapy, should be considered for women who experience vaginal recurrence of endometrial cancer.
\end{abstract}

Key words: endometrial cancer, high-dose-rate brachytherapy, vaginal recurrence.

\section{Purpose}

Endometrial cancer is the second most frequent gynecologic malignancy in Japan [1]. Standard management in Japan includes surgery followed by postoperative adjuvant chemotherapy in high-risk patients [2,3]. In contrast, surgery followed by postoperative radiotherapy is common in Europe and the United States [4]. The most common site of endometrial carcinoma recurrence is the pelvis, mostly in the vagina $[5,6,7,8]$. The optimal treatment for vaginal recurrence of endometrial cancer remains undetermined. To our knowledge, fewer studies have analyzed the effects of high-dose-rate (HDR) brachytherapy than of low-dose-rate (LDR) brachytherapy. Until 2008, standard treatment for vaginal recurrence of gynecologic carcinoma in our institution consisted of intracavitary brachytherapy (ICBT) with or without external beam radiotherapy (EBRT). Subsequently, our institution introduced interstitial brachytherapy (ISBT) for thick tumors that cannot be adequately treated by ICBT. This study investigated the outcomes of HDR brachytherapy (ICBT or ISBT), with or without EBRT, for women with vaginal recurrence of endometrial carcinoma, and analyzed factors prognostic of clinical outcomes.

\section{Material and methods}

\section{Patients}

The medical records of all patients treated with HDR brachytherapy for initial recurrence after definitive sur- 
gery of endometrial cancer in the vagina without pelvic nodal involvement at our institution from 1992 to 2014 were retrospectively reviewed. All patients had undergone hysterectomy and bilateral salpingo-oophorectomy, with or without lymph node assessment. Recurrence was confirmed pathologically by biopsy or cytology. Patients with distant metastases at the time of first relapse were excluded. The initial stage was determined according to the International Federation of Gynecology and Obstetrics (FIGO) classification, revised in 2009 [9].

\section{Brachytherapy}

Prior to 2008, patients underwent ICBT alone, with or without EBRT, with the latter performed at the discretion of the attending physician. After 2008, we introduced a HDR ISBT technique, as described $[10,11,12]$, with or without EBRT. Patients with tumors $>5 \mathrm{~mm}$ thick were treated with ISBT, whereas those with tumors $\leq 5 \mathrm{~mm}$ thick were treated with ICBT with or without EBRT after 2008. High-dose-rate brachytherapy was performed using a MicroSelectron HDR ${ }^{\mathrm{TM}}$ (Nucletron, an Elekta company, Elekta AB, Stockholm, Sweden). Before 2008, ICBT was planned using orthogonal two-dimensional images. After 2008, both ICBT and ISBT were planned by computed tomography (CT)-guided planning. Before 2008, patients with recurrent tumor invading paracolpial tissues underwent EBRT followed by ICBT. When recurrences were located in the upper and middle thirds of the vagina, clinical target volume (CTV) for both ICBT and ISBT was the gross tumor volume (GTV) and normal vaginal mucosa $2 \mathrm{~cm}$ caudal to the GTV. When recurrences involved the distal third of the vagina, the entire vagina was included in the CTV. In both orthogonal radiograph-based plans and the CT-based three-dimensional planned ICBT, the prescribed doses were administered to the vaginal wall at a $5 \mathrm{~mm}$ depth from the vaginal surface. A cylinder applicator was used for ICBT, whereas plastic applicators with handmade vaginal mold, a Syed-Neblett perineal template (Best Medical International, Inc., Springfield, VA, USA) or a cylinder was used for ISBT. The minimum dose covering $90 \%$ of the CTV $\left(\mathrm{CTV} \mathrm{D}_{90}\right)$ in the equivalent dose in $2 \mathrm{~Gy}$ fractions $\left(\mathrm{EQD}_{2}\right)$ was used as the prescribed dose of ISBT. After 2008, CTV was defined based on the CT image (Aquilion LG, Toshiba, Tokyo, Japan) taken after applicator insertion, with the patient in the lithotomy position, with information derived from gynecological examination, intra-operative transrectal ultrasound images, and the latest magnetic resonance imaging, if available. Interstitial brachytherapy was delivered twice daily with each fraction 6 hours apart. The cumulative doses were calculated on the basis of $\mathrm{EQD}_{2}$, which were calculated in 2 Gy equivalents using the equation derived from the linear-quadratic model [13]:

$$
\mathrm{EQD} 2=\mathrm{D}^{*}[(\mathrm{~d}+\alpha / \beta) /(2 \mathrm{~Gy}+\alpha / \beta)],
$$

in which $D$ represents total dose, $d$ represents fraction dose, $\alpha$ represents linear (first-order, dose-dependent) component of cell killing, $\beta$ represents quadratic (second-order, dose-dependent) component of cell kill- ing (more reparable), and $\alpha / \beta$ represents the dose at which both components of cell killing are equal. When calculating doses of tumor and normal tissue, $\alpha / \beta$ was assumed to be $10 \mathrm{~Gy}$ and $3 \mathrm{~Gy}$, respectively. The dose from the pelvic side wall boost with central shield was not included.

\section{Analysis}

Overall survival (OS), local control (LC), and progression-free survival (PFS) rates were defined as the duration from the first day of whole radiotherapy, until the date of the documented event, censored at the time of last follow-up. Local recurrence was defined as pelvic relapse, including the vagina and regional pelvic lymph nodes. Local recurrence and/or distant metastasis after radiotherapy was diagnosed by gynecological examination, cytology, and CT and/or magnetic resonance imaging (MRI). OS, LC, and PFS rates were estimated by the Kaplan-Meier method and compared using log-rank test. Analyzed variables included tumor size $(<25 \mathrm{~mm}$ vs. $\geq 25 \mathrm{~mm}$ ), initial FIGO stage, recurrent site (upper and middle vs. lower third of the vagina), initial histologic grade (grade $1 / 2$, grade 3 ), time interval between surgery and first recurrence ( $<1$ year vs. $\geq 1$ year), time interval between the diagnosis of first recurrence and the start of radiotherapy, radiotherapy schedule (EBRT + brachytherapy vs. brachytherapy alone), brachytherapy modality (ICBT vs. ISBT), and cumulative radiotherapy dose (<65 Gy vs. $\geq 65$ Gy). All tests of hypotheses were conducted at an $\alpha$ level of 0.05 with $95 \%$ confidence intervals. All statistical analyses were performed using SPSS Statistics version 21.0 (SAS Institute, Tokyo, Japan).

\section{Adverse effects}

Acute toxicities were graded according to the Common Terminology Criteria for Adverse Events, version 4. Late morbidities were assessed using the European Organization for Research and Treatment of Cancer/Radiation Therapy Oncology Group Late Effects Normal Tissue Task Force, Subjective, Objective, Management, and Analytic scale, revised in $1995[14,15,16]$. Adverse effects were reported as absolute number of events for grade 0 (no side effects) to grade 4 (worst side effects) morbidities. When reirradiation was performed for the second relapse after the brachytherapy for the first recurrence, adverse effects were estimated by the date of the reirradiation.

Our study was approved by the institutional ethical review board and was performed in accordance with the ethical standards laid down in the 1964 Declaration of Helsinki and its later amendments.

\section{Results}

Thirty-seven patients were eligible for our study; their demographic and clinical characteristics at the time of initial treatment are shown in Table 1. Median age at first recurrence was 67 years (range: $43-84$ years). Information about initial FIGO stage was not available for one patient, because she had undergone surgery in another country and was referred to our institution without stage 
Table 1. Patients' demographic and clinical characteristics at the time of primary treatment $(n=37)$

\begin{tabular}{lc} 
Factor & Patients $(n)$ \\
\hline Initial FIGO stage & $22(59.5 \%)$ \\
\hline 1 & $6(16.2 \%)$ \\
\hline ND & $8(21.6 \%)$ \\
\hline Histology & $1(2.7 \%)$ \\
\hline Endometrioid adenocarcinoma & $30(81.1 \%)$ \\
\hline Serous adenocarcinoma & $2(5.4 \%)$ \\
\hline Clear cell adenocarcinoma & $2(5.4 \%)$ \\
\hline Unclassified adenocarcinoma & $2(5.4 \%)$ \\
\hline Unknown & $1(2.7 \%)$ \\
\hline Postoperative adjuvant therapy & $1(2.7 \%)$ \\
\hline Radiotherapy & $11(29.7 \%)$ \\
\hline Chemotherapy & $25(67.6 \%)$ \\
\hline None
\end{tabular}

FIGO - International Federation of Gynecology and Obstetrics, ND - no data

information. Initial postoperative treatment included radiotherapy (EBRT) in one patient (48.6 Gy in 27 fractions) and chemotherapy in 11 , including four who received adriamycin and cisplatin, four who received paclitaxel and carboplatin, and three who received cyclophosphamide, adriamycin, and cisplatin. The interval time from surgery to the first recurrence ranged 2 to 183 months (median - 15 months, mean - 27 months) (Table 2). Median tumor size at initial examination for first recurrence was $12 \mathrm{~mm}$ (range, 2-43 mm), $2 \mathrm{~mm}$ in the ICBT group, and $26 \mathrm{~mm}$ in the ISBT group $(p<0.001)$. In 30 patients, tumors were involved in the upper and middle thirds of the vagina. In the other seven patients, tumors were localized to the distal third of the vagina. The interval time from the date of diagnosis of first recurrence to the start of radiotherapy ranged from 0.1 to 16.4 months (median - 1.3 months, mean - 2.7 months) (Table 3). After the diagnosis of first recurrence, three patients received chemotherapy, one underwent tumor reduction surgery followed by chemotherapy, and one underwent surgical debulking with intention before radiotherapy. Twentythree patients received ICBT and 14 received ISBT (Figure 1). EBRT to the pelvic side wall was delivered before or after brachytherapy to 26 patients, with a median dose of 50 Gy (range, 30-50 Gy). Of these 26 patients, 24 received 50 Gy EBRT, with 22 of these 24 adopting a central shield up to a median dose of 40 Gy (range, 20-40 Gy). The median cumulative $\mathrm{EQD}_{2}$ of EBRT and brachytherapy for vaginal recurrence was 62 Gy (range, 40-80 Gy). Fourteen patients received ICBT using orthogonal radiograph-based plans, whereas the other
Table 2. Patients' demographic and clinical characteristics at the time of first recurrence $(n=37)$ Factor

Patients $(n)$

\begin{tabular}{|c|c|}
\hline \multicolumn{2}{|l|}{ Age (years) } \\
\hline Median & 67 \\
\hline Range & $43-84$ \\
\hline \multicolumn{2}{|c|}{ Time interval from surgery to recurrence (months) } \\
\hline Median & 15 \\
\hline Range & $2-183$ \\
\hline \multicolumn{2}{|l|}{ Site of recurrence } \\
\hline Upper 2/3 vagina & $30(81.1 \%)$ \\
\hline Lower $1 / 3$ vagina & $7(18.9 \%)$ \\
\hline \multicolumn{2}{|l|}{ Tumor size $(\mathrm{mm})$} \\
\hline Median & 12 \\
\hline Range & $2-43$ \\
\hline
\end{tabular}

Table 3. Treatment for recurrence Treatment for recurrence

Patients ( $n$ )

Time interval from diagnosis of recurrence to radiotherapy (months)

$$
\text { Median (range) } 1.3(0.1-16.3)
$$

Treatment prior to radiotherapy

\begin{tabular}{ll}
\hline Chemotherapy & $3(8.1 \%)$ \\
\hline Surgery & $1(2.7 \%)$ \\
\hline Surgery + chemotherapy & $1(2.7 \%)$ \\
\hline
\end{tabular}

Radiotherapy modality

\begin{tabular}{lc}
\hline ICBT alone & $9(24.35 \%)$ \\
\hline EBRT + ICBT & $14(37.8 \%)$ \\
\hline ISBT alone & $2(5.4 \%)$ \\
\hline EBRT + ISBT & $12(32.4 \%)$
\end{tabular}

\section{EBRT (Gy)}

\begin{tabular}{lc}
\hline Median dose (range) & $50(30-50)$ \\
\hline Median number of fractions (range) & $25(15-25)$ \\
\hline
\end{tabular}

\begin{tabular}{lc}
\hline Brachytherapy (Gy) & \\
\hline Median dose (range) & $24(12-54)$ \\
\hline Median number of fractions (range) & $4(2-9)$ \\
\hline
\end{tabular}

Cumulative radiotherapy dose (Gy in $\mathrm{EQD}_{2}$ )

Median dose (range)

ICBT - intracavitary brachytherapy, ISBT - interstitial brachytherapy, $E B R T$ - external beam radiotherapy, $E Q D_{2}$ - equivalent dose in $2 \mathrm{~Gy}$ 

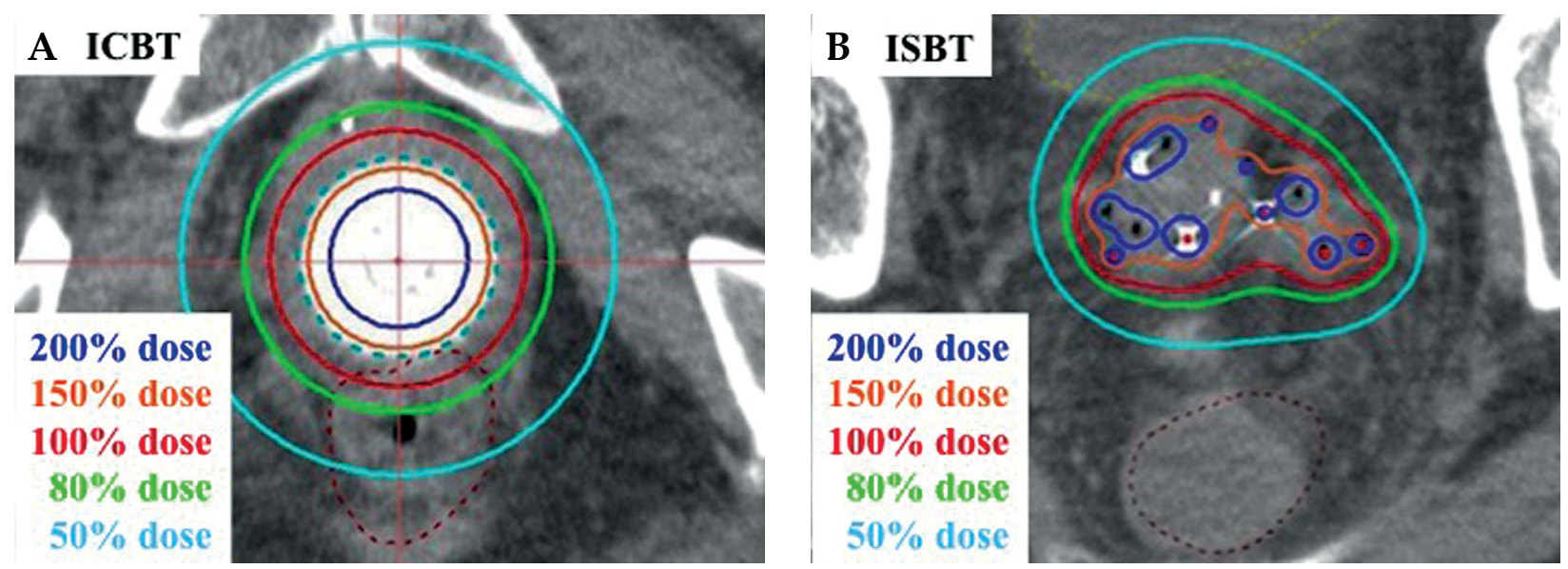

Fig. 1. Dose distributions of two brachytherapy modalities: (A) intracavitary brachytherapy (ICBT), and (B) interstitial brachytherapy (ISBT)

23 received ICBT or ISBT according to CT-based threedimensional planning. The median cumulative $\mathrm{EQD}_{2}$ to the tumors was 56 Gy in the ICBT group and 70 Gy in the ISBT group $(p<0.001)$.

\section{Overall survival and local control}

The median follow-up time was 48 months (range, 6-225 months). The 4-year OS and LC rates were $81.0 \%$ and $77.9 \%$, respectively (Figure 2). Local recurrence in the vagina alone was observed in seven patients, whereas none developed distant metastases simultaneously with local recurrence. Of 7 , three patients received reirradiation for

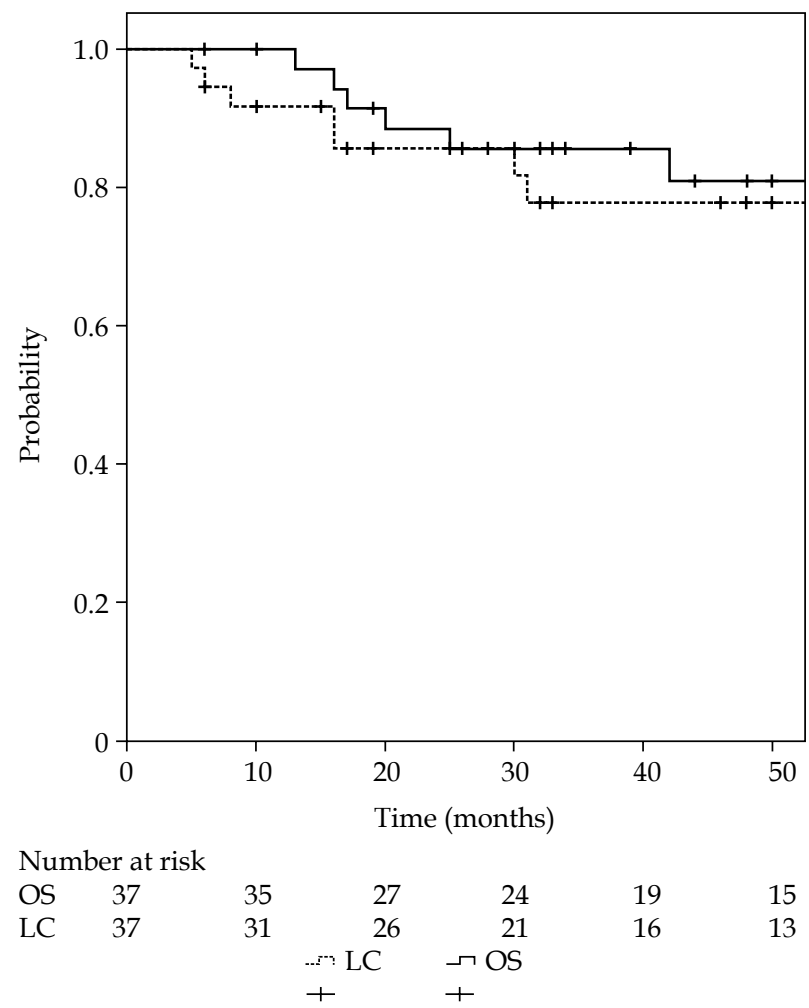

Fig. 2. Kaplan-Meier analysis of overall survival (solid line) and local control (dashed line) in the entire patient cohort the second vaginal relapse. At the last follow-up, 8 patients had died. Of 8 , seven died of distant metastases of endometrial carcinoma. The characteristics of patients in the ICBT and ISBT groups are shown in Table 4. The 4-year OS and LC rates were $89.1 \%$ and $75.3 \%$, respectively, in the ICBT group, and $68.4 \%$ and $85.7 \%$, respectively, in ISBT group, with no significant between group differences in OS $(p=0.11$, Figure 3$)$ and LC ( $p=0.82$, Figure 4$)$ rates.

Table 4. Demographic and clinical features by brachytherapy modality

$$
\text { ICBT }(n=23) \quad \text { ISBT }(n=14) \quad p \text { value }
$$

\begin{tabular}{|c|c|c|c|}
\hline \multicolumn{4}{|c|}{ Age at time of first recurrence } \\
\hline Median & 65 & 68.5 & 0.77 \\
\hline Initial FIGO stage & & & 0.50 \\
\hline I & 15 & 7 & \\
\hline II, III, IV & 8 & 6 & \\
\hline Initial histologic grade & & & 0.11 \\
\hline 1,2 & 21 & 10 & \\
\hline 3 & 2 & 4 & \\
\hline Site of recurrence & & & 0.08 \\
\hline Upper 2/3 vagina & 21 & 9 & \\
\hline Lower $1 / 3$ vagina & 2 & 5 & \\
\hline \multicolumn{4}{|l|}{ Tumor size (mm) } \\
\hline Median (range) & $2(2-25)$ & $26(2-43)$ & $<0.001$ \\
\hline \multicolumn{4}{|l|}{ EBRT for tumor (Gy) } \\
\hline Median (range) & $30(0-50)$ & $30(0-50)$ & 0.41 \\
\hline \multicolumn{4}{|l|}{ Brachytherapy (Gy) } \\
\hline Median (range) & $24(12-42)$ & $24(12-54)$ & 0.43 \\
\hline
\end{tabular}

ICBT - intracavitary brachytherapy, ISBT - interstitial brachytherapy, FIGO - International Federation of Gynecology and Obstetrics, EBRT - external beam radiotherapy 


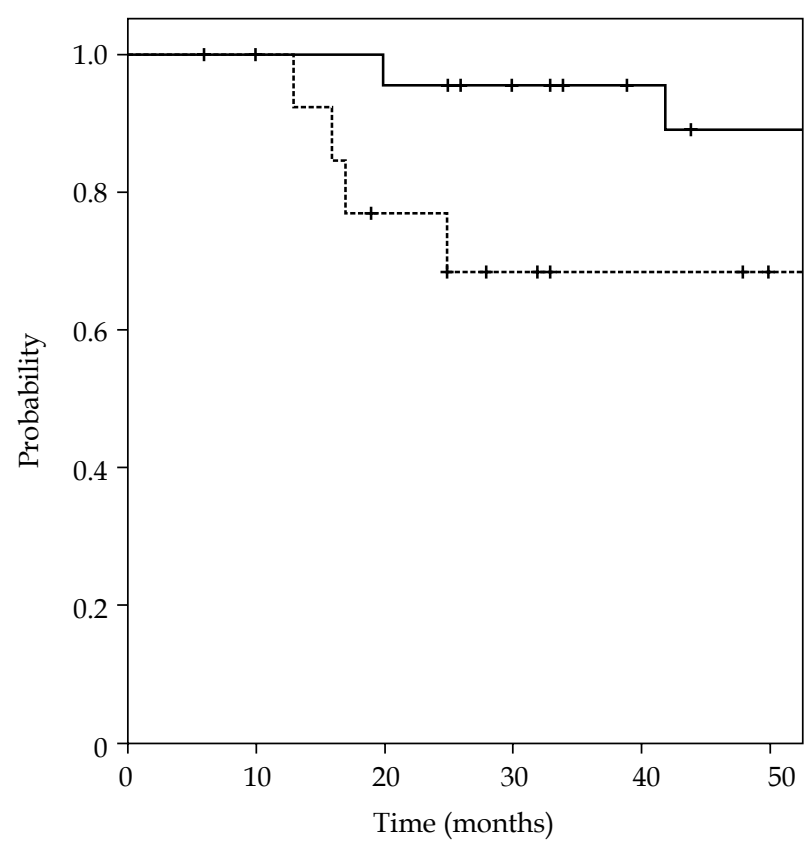

Number at risk

$\begin{array}{lllrccc}\text { ICBT } & 23 & 22 & 21 & 18 & 15 & 13 \\ \text { ISBT } & 14 & 13 & 9 & 6 & 4 & 2 \\ & & & & & \end{array}$

Fig. 3. Kaplan-Meier analysis of overall survival in patients who received intracavitary brachytherapy (ICBT, solid line) and interstitial brachytherapy (ISBT, dashed line)

Univariate analysis showed that the time interval between the diagnosis of first recurrence and the start of radiotherapy ( $<3$ months, $\geq 3$ months) significantly affected LC ( $p=0.045$, Figure 5$)$, but not OS $(p=0.41)$. Of 6 patients with time intervals $\geq 3$ months, three received chemotherapy and one underwent surgery followed by chemotherapy in other hospitals. Tumor size and initial histologic grade were significantly prognostic for OS. The 4-year OS rate was significantly higher in patients with tumors $<25 \mathrm{~mm}$ than $\geq 25 \mathrm{~mm}(86.9 \%$ vs. $57.1 \%, p=0.004)$ and in those with initial histologic grade $1 / 2$ than those with grade $3(88.0 \%$ vs. $40.0 \%, p=0.002)$.

Initial FIGO stage, site of recurrence, interval time between surgery and first recurrence, radiotherapy schedule, brachytherapy modality, and cumulative radiotherapy dose (<65 Gy, $\geq 65 \mathrm{~Gy}$ ) were not significantly predictive of OS or LC.

\section{Progression-free survival}

The 4-year PFS rate was $56.8 \%$. Nine patients experienced distant metastases as the second relapse. Eight developed in lung and 1 in lung, bone, and paraaortic lymph node.

The interval time between the diagnosis of the first recurrence and start of radiotherapy was significantly associated with PFS rate $(p=0.023)$. The 4 -year PFS rate was significantly higher in patients with short $(<3$ months) than long ( $\geq 3$ months) intervals $(65.4 \%$ vs. $25.0 \%$, $p=0.023)$. PFS rate was not significantly associated with tumor size, initial FIGO stage, site of recurrence, histologic

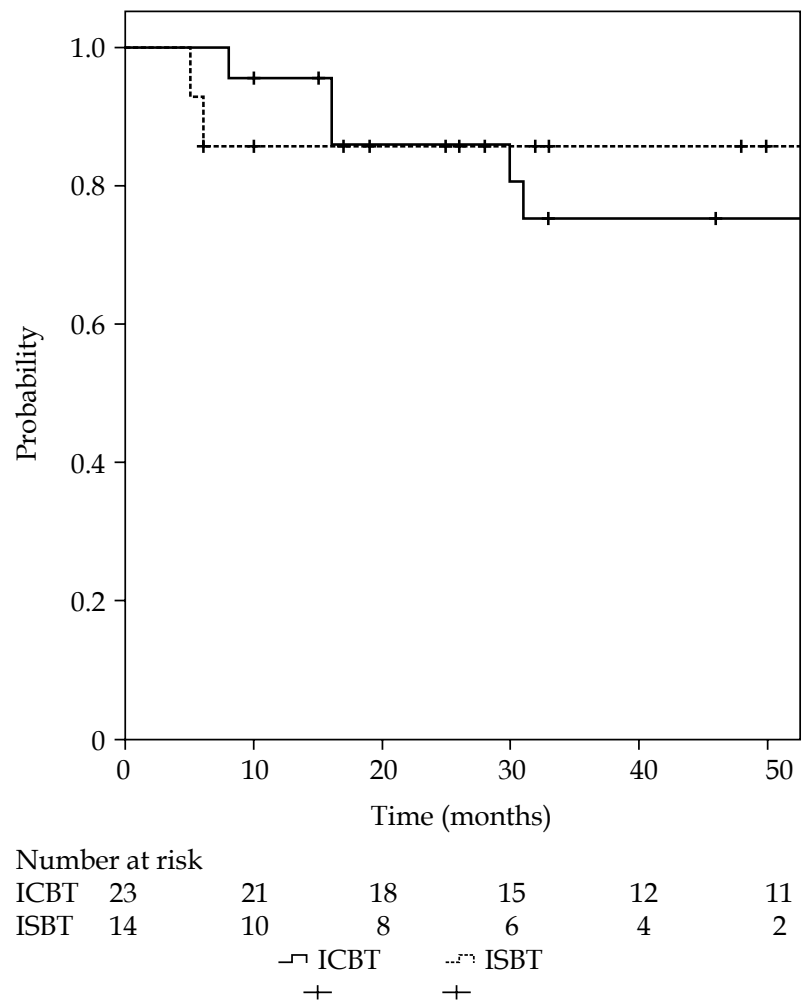

Fig. 4. Kaplan-Meier analysis of local control in patients who received intracavitary brachytherapy (ICBT, solid line) and interstitial brachytherapy (ISBT, dashed line)

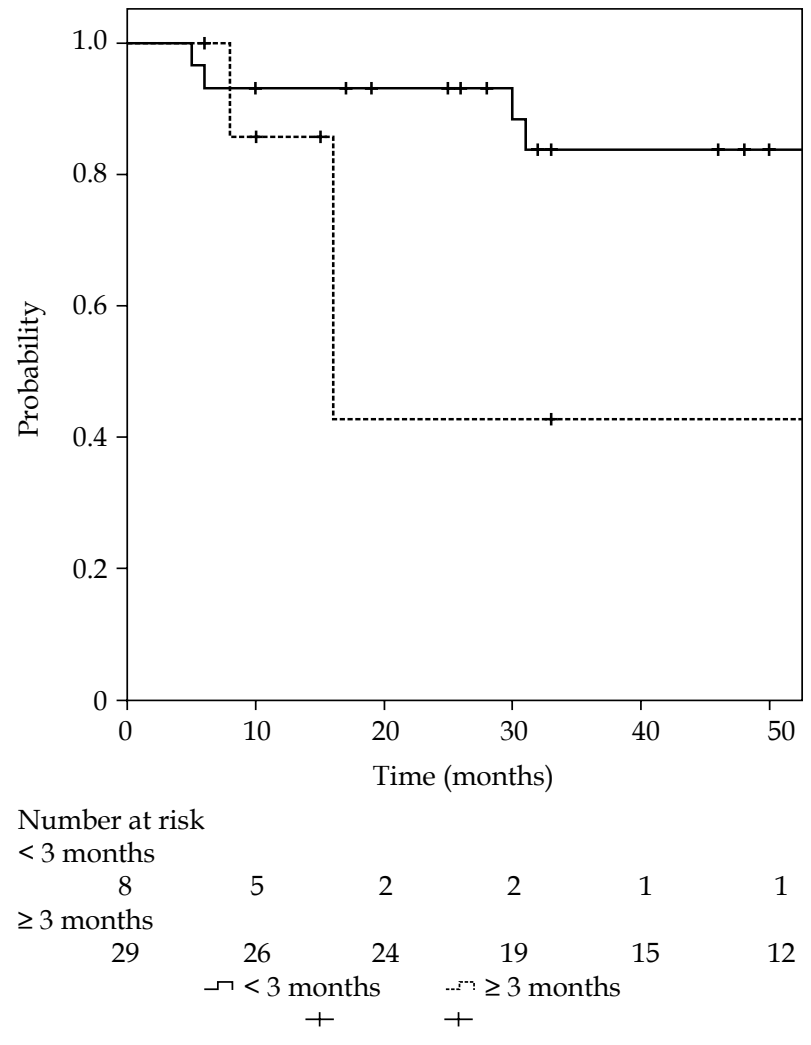

Fig. 5. Kaplan-Meier analysis of local control in patients who underwent radiotherapy $<3$ months (solid line) and $\geq 3$ months (dashed line) after diagnosis of first recurrence 
grade, time interval between surgery and first recurrence, radiotherapy schedule, brachytherapy modality, and cumulative radiotherapy dose.

\section{Adverse effects}

No patient experienced grade 3 or higher acute toxicity. Late radiotherapy associated complications occurred in 15 patients. Two patients experienced grade 1 vaginal ulceration (superficial $\leq 1 \mathrm{~cm}^{2}$ ), whereas none developed grade 2 vaginal ulceration (superficial $>1 \mathrm{~cm}^{2}$ ). Two patients, who received radiation doses to the rectum of $63.7 \mathrm{~Gy}$ and $70.2 \mathrm{~Gy}$, developed grade 2 rectal bleeding (occasional $>2$ /week). Four patients experienced late grade 2 hematuria (intermittent macroscopic), whereas none experienced grade 3 or higher late toxicity.

\section{Discussion}

This study showed that salvage HDR brachytherapy, with or without EBRT, resulted in favorable OS and LC for patients with vaginal recurrence of endometrial cancer. At 4-years, OS, LC, and PFS rates were $81.0 \%, 77.9 \%$, and $56.8 \%$, respectively. This LC rate compared favorably with that of other large reported series, including one report showing 5-year LC rate of 75\% [17]. Long-term follow-up of 20 patients showed that the 10 -year cumulative OS and LC rates were $48 \%$ and $74 \%$, respectively [18], with estimated 4-year OS and LC rates of approximately $80 \%$ and $74 \%$, respectively. Variations in OS and LC were likely due to the small numbers of patients in these studies and differences in radiation modalities. The 5-year LC rate in 22 patients who received HDR brachytherapy was reported to be $100 \%$ [19]. The different LC rates reported in our study may be attributed to the proportion (82\% vs. $38 \%$ ) of women who received ICBT and EBRT.

OS and LC rates did not differ significantly in the two brachytherapy groups (ICBT vs. ISBT), although larger tumors were included in the ISBT group. Therefore, the disadvantage of larger tumor volume can be overcome by applying ISBT. The 4-year OS and LC in ISBT group were $68.4 \%$ and $85.7 \%$, respectively. Few previous reports have evaluated ISBT for vaginal recurrence of endometrial carcinoma. One reported 5-year OS and LC rates of $56 \%$ and $70 \%$, respectively [20]. Use of CT or MRI guided ISBT in patients without prior radiotherapy resulted in a 2-year LC rate of $96 \%$ [21]. Our results were not inferior to these findings. Interstitial brachytherapy had clinical significance for relatively large tumors with paracolpial extension or located more than $5 \mathrm{~mm}$ from the vaginal membrane.

Our study showed that the interval time between the diagnosis of first recurrence and the start of radiotherapy (<3 months vs. $\geq 3$ months) was significantly prognostic of LC. Most patients (66.7\%) with intervals of $\geq 3$ months initially received chemotherapy or surgery for first relapse, delaying the start of salvage radiation treatment. Postoperative brachytherapy with or without EBRT of uterine corpus has been used to treat women in Western countries $[22,23]$. In contrast, a study by the Japanese Gynecologic Oncologic Group (JGOG2033) showed that OS rate in patients at high to intermediate risk was high- er in those who received postoperative chemotherapy than postoperative pelvic radiotherapy [2]. Therefore, most of these patients in Japan with first relapse undergo radiotherapy without concern about reirradiation in Japan, where those with risk factors after hysterectomy are more likely to receive adjuvant chemotherapy than radiotherapy. Because postoperative therapies differ between Japan and Western countries, our results could not be readily compared with those of Western countries. Pelvic exenteration is also a choice of treatment for recurrence confined to the vagina or the pelvis alone [4], whereas surgery is effective when the recurrence can be resected completely. Although our study did not directly compare radiotherapy with chemotherapy or surgery for first vaginal relapse, the high local control rate obtained with early brachytherapy, with or without EBRT suggests that this modality remains an optimal therapy for isolated vaginal recurrence of endometrial carcinoma, without the need for surgery or chemotherapy.

We found that tumor size $(<25 \mathrm{~mm}$ vs. $\geq 25 \mathrm{~mm})$ and initial histologic grade (grade $1 / 2$ vs. grade 3 ) had an impact on OS rate. Because most patients (87.5\%) who died of endometrial carcinoma had distant metastases, control of distant metastases is mandatory for patients with large recurrent tumors. Adjuvant chemotherapy may be an option for patients with tumor size $\geq 25 \mathrm{~mm}$. Another study reported that tumor size $(\leq 25 \mathrm{~mm}$ vs. $>25 \mathrm{~mm}$ ), extra-vaginal extension, and the stage of initial disease had an impact on OS in 25 patients who received LDR brachytherapy with or without EBRT [24]. Moreover, multivariate analysis in 50 patients, two of whom received EBRT alone, eight of whom received brachytherapy (LDR or HDR) alone, and 40 of whom received both, found that tumor size, histologic grade, and age were significant predictors of OS [25]. A third study reported that histologic grade (grade $1 / 2$ vs. grade 3 ) and therapy (EBRT plus brachytherapy versus single-modality therapy) were significant predictors of OS [17]. Moreover, univariate analysis of 40 patients who received HDR brachytherapy found that FIGO grade, DNA ploidy, and risk group were significant predictors of OS [26].

None of our patients experienced grade 3 or higher late complication. In contrast, other studies have reported that $10 \%$ and $18 \%$ of patients experienced grade $3-4$ gastrointestinal complications, whereas none experienced grade 3-4 genitourinary complications $[19,25]$. A larger series reported that 8 of 91 patients $(9 \%)$ had grade 4 late complications (fistula, bowel obstruction, gastrointestinal bleeding) [17]. Compared with these other reports, we found that gastrointestinal toxicities were less frequent and genitourinary toxicities were of similar frequency.

Our study had several limitations, including its retrospective design, small number of patients treated at a single institution, and the clinical heterogeneity of these patients. Moreover, radiotherapy modalities and prescribed doses were varied.

\section{Conclusions}

Salvage radiotherapy, consisting of HDR brachytherapy with or without EBRT yielded favorable LC and OS 
rates for patients with vaginal recurrence of endometrial carcinoma, with acceptable morbidity. Early salvage HDR brachytherapy was optimal treatment. LC and OS rates were similar in patients who underwent ISBT for larger sized tumors, and ICBT for superficial and smaller tumors.

\section{Acknowledgements}

This study was financially supported in part by the Practical Research for Innovative Caner Control from Japan Agency for Medical Research and development, AMED (15ck0106039h0002).

\section{Disclosure}

Authors report no conflict of interest.

\section{References}

1. Hori M, Matsuda T, Shibata A et al. Cancer incidence and incidence rates in Japan in 2009: a study of 32 population-based cancer registries for the Monitoring of Cancer Incidence in Japan (MCIJ) project. Jpn J Clin Oncol 2015; 45: 884-891.

2. Susumu N, Sagae S, Udagawa $Y$ et al. Randomized phase III trial of pelvic radiotherapy versus cisplatin-based combined chemotherapy in patients with intermediate- and high-risk endometrial cancer: a Japanese Gynecologic Oncology Group study. Gynecol Oncol 2008; 108: 226-233.

3. Ebina Y, Katabuchi H, Mikami M et al. Japan Society of Gynecologic Oncology guidelines 2013 for the treatment of uterine body neoplasms. Int J Clin Oncol 2016; 21: 419-434.

4. National Comprehensive Cancer Network Guidelines Uterine Neoplasms Version 1 2017; https://www.nccn.org/professionals/physician_gls/f_guidelines.asp.

5. Keys HM, Roberts JA, Brunetto VL et al. A phase III trial of surgery with or without adjunctive external pelvic radiation therapy in intermediate risk endometrial adenocarcinoma: a Gynecologic Oncology Group study. Gynecol Oncol 2004; 92: 744-751.

6. Chadha M, Nanavati PJ, Liu P et al. Patterns of failure in endometrial carcinoma stage IB grade 3 and IC patients treated with postoperative vaginal vault brachytherapy. Gynecol Oncol 1999; 75: 103-107.

7. Greven KM. Tailoring radiation to the extent of disease for uterine-confined endometrial cancer. Semin Radiat Oncol 2000; 10: 29-35.

8. Creutzberg CL, van Putten WLJ, Koper PCM et al. Surgery and postoperative radiotherapy versus surgery alone for patients with stage-1 endometrial carcinoma: multicentre randomized trial. PORTEC Study Group. Post-Operative Radiation Therapy in Endometrial Carcinoma. Lancet 2000; 355: 1404-1411.

9. Creasman W. Revised FIGO staging for carcinoma of the endometrium. Int J Gynaecol Obstet 2009; 105: 109.

10. Itami J, Hara R, Kozuka T et al. Transperineal high-dose-rate interstitial radiation therapy in the management of gynecologic malignancies. Strahlenther Onkol 2003; 179: 737-741.

11. Murakami N, Kasamatsu T, Sumi M et al. Vaginal tolerance of CT based image-guided high-dose rate interstitial brachytherapy for gynecological malignancies. Radiat Oncol 2014; 9: 31.

12. Murakami N, Kato T, Miyamoto $Y$ et al. Salvage high-doserate interstitial brachytherapy for pelvic recurrent cervical carcinoma after hysterectomy. Anticancer Res 2016; 36: 2413-2421.

13. Withers HR, Thames HD Jr, Peters LJ. A new isoeffect curve for change in dose per fraction. Radiother Oncol 1983; 1: 187-191.
14. Coia LR, Myerson RJ, Tepper JE. Late effects of radiation therapy on the gastrointestinal tract. Int J Radiat Oncol Biol Phys 1995; 31: 1213-1236.

15. Grigsby PW, Russell A, Bruner D et al. Late injury of cancer therapy on the female reproductive tract. Int J Radiat Oncol Biol Phys 1995; 31: 1281-1299.

16. Marks LB, Carroll PR, Dugan TC et al. The response of the urinary bladder, urethra, and ureter to radiation and chemotherapy. Int J Radiat Oncol Biol Phys 1995; 31: 1257-1280.

17. Jhingran A, Burke TW, Eifel PJ. Definitive radiotherapy for patients with isolated vaginal recurrence of endometrial carcinoma after hysterectomy. Int J Radiat Oncol Biol Phys 2003; 56: 1366-1372.

18. Pai HH, Souhami L, Clark BG et al. Isolated vaginal recurrences in endometrial carcinoma: treatment results using highdose-rate intracavitary brachytherapy and external beam radiotherapy. Gynecol Oncol 1997; 66: 300-307.

19. Petignat $P$, Jolicoeur $M$, Alobaid A et al. Salvage treatment with high-dose-rate brachytherapy for isolated vaginal endometrial cancer recurrence. Gynecol Oncol 2006; 101: 445-449.

20. Charra C, Roy P, Coquard R et al. Outcome of treatment of upper third vaginal recurrences of cervical and endometrial carcinomas with interstitial brachytherapy. Int J Radiat Oncol Biol Phys 1998; 40: 421-426.

21. Lee LJ, Damato AL, Viswanathan AN. Clinical outcomes following 3D image-guided brachytherapy for vaginal recurrence of endometrial cancer. Gynecol Oncol 2013; 131: 586-592.

22. Huddleston A, Zhen S, Qi L et al. The impact of a vaginal brachytherapy boost to pelvic radiation in stage III endometrial cancer. J Contemp Brachytherapy 2015; 7: 122-127.

23. Thompson SR, Delaney GP, Gabriel GS et al. Patterns of care study of brachytherapy in New South Wales: malignancies of the uterine corpus. J Contemp Brachytherapy 2015; 7: 224-230.

24. Hasbini A, Haie-Meder C, Morice P et al. Outcome after salvage radiotherapy (brachytherapy $+/$ - external) in patients with a vaginal recurrence from endometrial carcinomas. Radiother Oncol 2002; 65: 23-28.

25. Lin LL, Grigsby PW, Powell MA et al. Definitive radiotherapy in the management of isolated vaginal recurrences of endometrial cancer. Int J Radiat Oncol Biol Phys 2005; 63: 500-504.

26. Sorbe B, Soderstrom K. Treatment of vaginal recurrences in endometrial carcinoma by high-dose-rate brachytherapy. Anticancer Res 2013; 33: 241-247. 